\title{
Age and gender differences in children's food preferences
}

\author{
Lucy J. Cooke* and Jane Wardle \\ Cancer Research UK Health Behaviour Unit, Department of Epidemiology and Public Health, University College London, WC1E 6BT UK \\ (Received 19 July 2004 - Revised 29 November 2004 - Accepted 30 November 2004)
}

\begin{abstract}
The present study was conducted to examine the developmental patterning of food preferences in a large sample of British schoolchildren and to investigate possible gender differences. Using a cross-sectional survey design, the study was carried out in three primary and three secondary schools in West London, UK. A total of 1291 children aged from 4 to 16 years completed a 115-item food preference questionnaire in class time, supervised by class teachers and assistants. Children indicated whether they had ever tried each item and, if so, how much they liked it. We observed age-related increases in the number of foods tried $(P<0.001)$, liked $(P<0.005)$ and disliked $(P<0.05)$. Controlling for the number of foods tried rendered the increase in dislikes non-significant and reversed the age effect on the number liked. Girls liked fruit $(P<0.05)$ and vegetables $(P<0.001)$ more than boys did; boys liked fatty and sugary foods $(P<0.005)$, meat $(P<0.001)$, processed meat products $(P<0.001)$ and eggs $(P<0.05)$ more than girls did. Some age differences were apparent in liking for categories of food, although the effects were not linear. Across ages and genders, children rated fatty and sugary foods most highly, although ratings for fruit were also high. Children's food preferences overall are not consistent with a healthy diet. Interventions should focus on increasing the familiarity, availability and accessibility of healthy foods and should be mindful of the need to target messages appropriately for boys who have less healthful food preferences than girls at all ages.
\end{abstract}

Child: Food preferences: Age differences: Gender differences

It is widely held that children 'eat what they like', and research has repeatedly shown that children's food preferences are highly predictive of their intake (Birch, 1979b; Drewnowski, 1997; Resnicow et al. 1997; Gibson et al. 1998; Baxter \& Thompson, 2002; PerezRodrigo et al. 2003). Unfortunately, the foods that children like most are rarely of high nutritional value. Even in France, where the majority of 9-11 year-old children still eat traditional 'family dinners', a recent study found that the self-reported 'Top 10' foods included French fries, chocolate, pizza, cake and ice cream (Bellisle et al. 2000). Similar items have appeared among the favourite foods of older French children (Ton Nu et al. 1996), American 2-8-year-olds (Skinner et al. 2002), German 10-14-year-olds (Diehl, 1999) and British 4-5-year-olds (Wardle et al. 2001). Equally consistent cross-culturally are children's dislikes, with vegetables featuring reliably among the least favoured foods (Ton $\mathrm{Nu}$ et al. 1996; Diehl, 1999; Skinner et al. 2002; Perez-Rodrigo et al. 2003). This pattern of preferences is consistent with the evidence for innate predispositions to prefer sweet tastes, to learn to prefer energy-dense foods and to dislike those which are sour or bitter (Birch, 1999).

Children's tendency to dislike new foods (neophobia) declines with age (Pelchat \& Pliner, 1995; Koivisto \& Sjoden, 1996), suggesting that their diets might diversify as they mature as a result of having tried a greater number of foods. In practice, many studies have found that children's eating habits are relatively stable over time (Lien et al. 2001; Sweeting et al. 1994; Skinner et al. 2002), while others have reported an age-related deterioration in dietary quality (Lytle et al. 2000), particularly in relation to fruit and vegetables. Possible explanations for these contradictory findings include different age groups of children studied and whether the outcome measure is liking or consumption.

Studies of age-related changes in food preferences are rare, and the findings of some are difficult to interpret. In a sample of 2-24-year-olds, Perez-Rodrigo et al. (2003) found few age differences in preferences for specific foods within food groups. However, information about the measures was sketchy, and it is not clear whether their results are comparable to those arising from the lengthy food lists usually employed.

A study of French 10-20-year-olds asked respondents to generate a maximum of ten liked and ten disliked foods and to recall at what age their food preferences (maximum 4-5) had changed (Ton Nu et al. 1996). Most participants reported that changes had occurred at around the age of 10, with negative changes (liking to disliking) taking place slightly earlier (mean age 10 years) and positive changes later (mean age 11 years). The authors conclude that a 'widening of food repertoire' occurs after puberty because of a reduction in neophobia together with an increase in autonomy concerning food and in eating out independently. Given the bluntness of the instrument and its reliance on participants' memory for events occurring up to 10 years previously, the interpretation of this small age difference should be approached with more caution. Nevertheless, taken together with the findings of a study of German 10-14-year-olds (Diehl, 1999) in which age was not 
significantly related to preferences, the emerging picture is one of relative stability in preferences after puberty.

In one of the few longitudinal studies in the field, Skinner and her colleagues analysed children's food preferences from the age of 2 to 8 together with an investigation of the factors related to preferences (Skinner et al. 2002). Across the 5 years of the study, the number of foods liked did not change significantly, although the number disliked and number tried increased. Longitudinal changes suggested that foods introduced after the age of 4 years were more likely to be disliked than liked. The differences between these findings and those from the older groups indicate a need for a study of children's food preferences that can span the transition from childhood to adolescence.

Findings concerning gender differences have been mixed. In the cohort of Lytle et al. (2000) cohort, developmental trends in consumption and eating patterns were very similar for boys and girls. Likewise, Perez-Rodrigo et al. (2003) reported few gender differences in the preferences of Spanish 2-24-year-olds. Among 4-5-year-old British children, girls liked vegetables more than boys did, but there were no gender differences in preferences in other food groups (Wardle et al. 2001). Boys were found to consume less fruit and like raw vegetables less than girls did in an ongoing study of French 9-11-year-olds (Le Bigot Macaux, 2001). Similar findings have been reported in American children and adolescents (Reynolds et al. 1999), German 10-14-year-olds (Diehl, 1999) and Norwegian 16-21year-olds (Lien et al. 2001), among others.

The present study examined the developmental patterning of food preferences in a large sample of British children from the first year of formal schooling (age 4-5 years) to the last year of compulsory education (age 16). On the basis of previous research, we expected some gender differences, and while the prediction of changes in preferences is highly complex, we hypothesised that number of foods tried would increase with age, with an associated increase in number of foods liked.

The study was exempt from the need for formal ethical approval according to the rules of University College London since it involved an entirely anonymous survey in which the investigators did not participate in data collection.

\section{Method}

\section{Design}

The study used a cross-sectional survey design to examine age and gender differences in the food preferences of British schoolchildren.

\section{Sample}

The target sample was all children in Years 1-11 registered at West London primary and secondary schools (roughly equivalent to elementary and junior high schools in North America).

\section{Procedure}

Schools in the London Borough of Hammersmith and Fulham were contacted by letter and invited to participate in a study of the development of children's food preferences. Three primary schools (all mixed-sex) and three secondary schools (one mixed-sex, one girls-only and one boys-only) agreed to take part. Questionnaires were distributed by teaching staff to all pupils in the primary schools $(n$ 840) and to two classes per year in the secondary schools ( $n$ 750). The questionnaires were completed during lessons or during private study periods, except for the youngest children (aged 4-7 years), who were asked to take their questionnaires home to complete with the help of their parents. Teachers read out standardised instructions to the pupils prior to filling in the questionnaires.

\section{Food preference questionnaire}

The Food Preference Questionnaire was based on one used by Wardle et al. (2001) in a study of 4-5-year-olds. Originally tested for reliability and validity when completed by parents (Pliner \& Pelchat, 1986), it has since been used as a selfreport measure for 9-11-year-olds (Gibson et al. 1998) and 4-22-year-olds (Nicklaus et al. 2004). A list of ninety-four common foods and beverages (Wardle et al. 2001) was amended for the present study in order to encompass a potentially wider range of foods available to older children. Drinks were excluded since preferences for drinks may be dictated by factors different from those for foods. For example, liking for carbonated drinks containing caffeine may be the result of negative reinforcement, linked to the removal of the negative effects of caffeine withdrawal in regular consumers (Garrett \& Griffiths, 1998).

The final questionnaire featured 115 food items including 'single' foods (e.g. apples), 'mixed' foods (e.g. lasagne) and 'condiments' (e.g. jam). Children were asked to indicate 'how much you like each food by ticking the appropriate box'. There were six response alternatives - 'never tried it', 'I hate it', 'I don't like it', 'it's OK', 'I like it' and 'I love it' - which were scored from 0 to 5 . Liking for a food was defined as a score of 4 or 5 , and disliking as a score of 1 or 2 . To facilitate understanding in younger children, a five-point 'faces scale' was added in questionnaires for the under-7s. In order to maximise participation rates, children were asked only for their age and gender and not required to give their name, ethnicity or any other socio-demographic information.

\section{Statistical analyses}

Data were analysed using SPSS version 10. (SPSS Inc., Chicago, IL, USA) Correlational analyses examined the relationship between the number of children having tried each food and the mean liking score of those who had tried it. One-sample $t$ tests were used to investigate whether average preference ratings differed significantly from indifference. Univariate ANOVA was used to examine age, gender and age-by-gender interaction effects on the number of foods tried, liked and disliked. The number of foods tried was entered as a covariate in further analyses to control for its effect on the number of foods available to be liked or disliked.

Foods were grouped into nine categories - 'Meat', 'Fish', 'Processed meat products', 'Eggs', 'Starchy staples', 'Fruit', 'Vegetables', 'Dairy', and 'Fatty \& sugary' - and the internal reliability of these groupings was assessed by Cronbach's alpha. A list of food items comprising each category is available from the authors on request. Foods tried by fewer than $75 \%$ of participants were not used in analyses of food groups. Further analyses investigated age and gender effects on mean liking scores for each category of foods. 


\section{Results}

Approximately $10 \%$ of children were absent from school on the day of the survey, and $8 \%$ failed to hand in their questionnaire, leaving an achieved sample of 1291 children, representing an $81.2 \%$ coverage rate of the 1590 children registered in the participating classes. Questionnaires with more than fifteen missing responses (representing more than one page) were excluded ( $n$ 59), which left data available for 1232 participants. Ages were 4 to 16 years, with $51 \%$ female, $46 \%$ male and $2 \%$ unspecified individuals. In order to examine age differences in food preference and familiarity, four age groupings were created: 'Infants', 4-7-year-olds (n 176); 'Juniors', 8-11-year-olds (n 291); 'Lower secondary', 11-13-year-olds attending secondary school ( $n$ 466); and 'Mid secondary' 14-16-year-olds ( $n$ 264). Thirty-five children omitted to record their age.

Children had tried an average of ninety-eight foods out of 115 . Across the whole sample, foods that were tried less often tended to be less liked and vice versa (Pearson correlation of percentage tried and mean liking score, $r 0.68, P<0.001)$. Univariate ANOVA revealed a strong increase with age in the number of foods tried $(F(3,1171)=75.34, P<0.001) \quad$ (Fig. 1). The number of foods liked $(F(3,1171)=4.50, P<0.005)$ and the number of foods disliked $(F(3,1171)=3.35, P<0.05)$ also increased with age. However, entering the number of foods tried as a covariate in further ANOVA reversed the pattern in number of foods liked such that, as a function of the number of foods tried, the number liked decreased with age $(F$ $(3,1167)=3 \cdot 27, P<0 \cdot 05)$ (Fig. 2$)$. The same procedure rendered the increase in foods disliked non-significant.

There were no significant gender effects for any of these three outcomes, but there was a significant age-by-gender interaction in the number of foods disliked $(F(3,1167)=2 \cdot 66, P<0.05)$, with younger boys disliking more foods than girls, whereas in the older groups this effect was reversed.

The ten most highly rated items were chocolate, pizza, ice cream, pasta, strawberries, chocolate biscuits, ice lollies, grapes, cakes and fruit sweets. The ten lowest rated foods included six vegetables (spinach, leeks, marrow, swede, sprouts and turnip) and some meats and meat substitutes (textured vegetable protein, soya meat, liver-sausage and liver).

Of the ninety-two foods tried by at least $75 \%$ of the sample, only ten were disliked on average (significantly below indifference by a one-sample $t$ test). These were processed cheese,

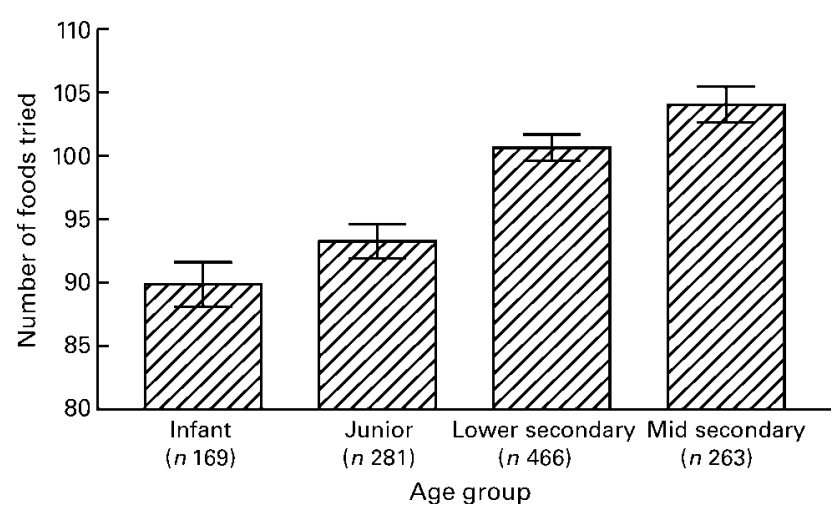

Fig. 1. Number of foods tried by different age groups. Values are means with $95 \% \mathrm{Cl}$ indicated by vertical bars. Univariate ANOVA showed a significant increase with age $(P<0.001)$.

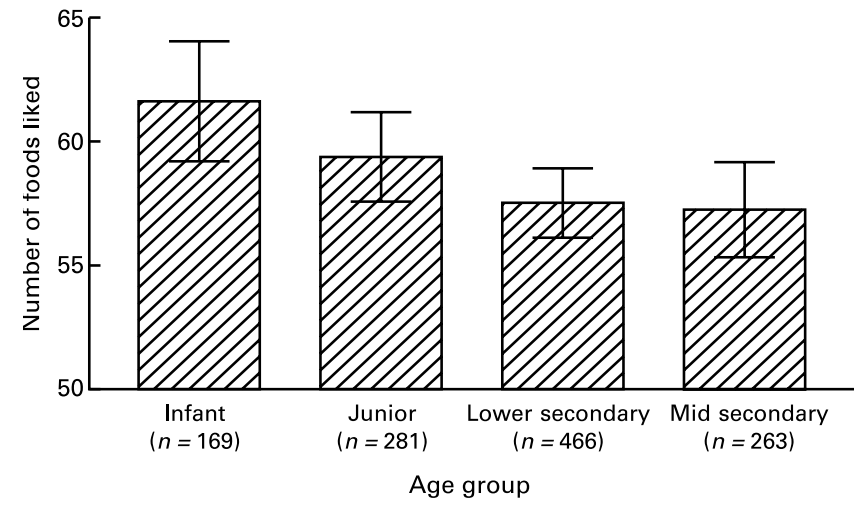

Fig. 2. Number of foods liked, adjusted for number of foods tried, by different age groups. Value are means with $95 \% \mathrm{Cl}$ indicated by vertical bars. Univariate ANOVA showed a significant decrease with age $(P<0.05)$.

cottage cheese, cabbage, cauliflower, mushrooms, onions, parsnips, sprouts, celery, and spinach. Among the twenty-three foods tried by fewer than $75 \%$ of the children, seventeen were significantly disliked by those who had tried them. All twentythree were omitted from further analyses of patterns of food preferences.

In order to examine the pattern of children's food preferences, the foods tried by at least $75 \%$ were grouped into categories of food type. These categories were fruit $(n 12)$, vegetables $(n 17)$, meat $(n 7)$, processed meat products $(n 7)$, starchy staples ( $n$ 12), eggs ( $n$ 3), fish (n 3), dairy $(n$ 7) and fatty \& sugary foods ( $n$ 19). Five items (nuts/nut dishes, soup, ketchup, salad dressing and gravy) were excluded at this stage since they could not be easily fitted into any one category. Category-based preference scales were produced for each participant by calculating the mean of the liking scores of the foods in each category. Cronbach's alpha and item means for each scale (for the total sample and for girls and boys separately) are given in Table 1.

Fatty/sugary foods were the most well-liked, followed by fruit, starchy staples, meat, processed meat products, eggs, fish, dairy foods and lastly vegetables. Liking for fruit and vegetables was higher for girls than boys $(F(1,1170)=4.22, P<0.05$ and $F(1,1171)=10 \cdot 56, P=0.001$, respectively). Liking for fatty $\&$ sugary foods, meat, processed meat products and eggs, on the other hand, was higher for boys $(F(1,1171)=9.46, P<0.005$; $F(1,1151)=40 \cdot 18, P<0 \cdot 001 ; F(1,1169)=25 \cdot 26, P<0 \cdot 001$; $F(1,1147)=3.98, P<0.05$, respectively). There were no gender differences in liking for foods in the fish, dairy or starchy staples categories.

There were age-related differences in preferences for foods in the fatty/sugary $(F(3,1171)=8.10, \quad P<0.001)$, fruit $(F$ $(3,1170)=3 \cdot 16, P<0 \cdot 05)$, fish $(F(3,1145)=4 \cdot 24, P<0 \cdot 05)$ and dairy $(F(3,1168)=4.77, P<0.005)$ categories (Table 2$)$. Liking for fruits and fatty \& sugary foods reached a peak at 8 11 years, whereas liking for fish and dairy foods was highest among the youngest children and declined thereafter.

Age-by-gender interaction effects on liking were observed in the processed meat products, eggs, fish and dairy foods categories only. For processed meat products, boys' liking increased with age whereas that of girls remained largely stable. Liking for eggs increased with boys' age but decreased with girls'. For dairy foods, boys' liking was seen to decline with age whereas that of girls fluctuated, and for fish items boys' liking remained static whereas girls' liking decreased with age. 
Table 1. Cronbach's alpha and mean liking* of items in each food category (Total sample and by gender)

\begin{tabular}{|c|c|c|c|c|c|c|c|c|}
\hline \multirow[b]{2}{*}{ Food category $(n)$} & \multirow[b]{2}{*}{ Scale alpha } & \multicolumn{2}{|c|}{ Total sample (n 1232) } & \multicolumn{2}{|c|}{ Girls ( $n$ 639) } & \multicolumn{2}{|c|}{ Boys ( $n$ 564) } & \multirow[b]{2}{*}{ Significance of difference } \\
\hline & & Mean & SD & Mean & SD & Mean & SD & \\
\hline Fruit (12) & 0.88 & $4 \cdot 18$ & 0.76 & $4 \cdot 22$ & 0.71 & $4 \cdot 13$ & 0.81 & $P<0.05$ \\
\hline Starchy staples (12) & 0.75 & 4.09 & 0.58 & 3.98 & 0.56 & 4.02 & 0.59 & NS \\
\hline Meat (7) & 0.77 & 3.72 & 0.79 & 3.57 & 0.80 & 3.90 & 0.79 & $P<0.001$ \\
\hline Processed meat (7) & 0.77 & 3.66 & 0.83 & 3.55 & 0.83 & $3 \cdot 81$ & 0.80 & $P<0.001$ \\
\hline Eggs (3) & 0.86 & 3.54 & $1 \cdot 26$ & 3.46 & $1 \cdot 22$ & $3 \cdot 64$ & $1 \cdot 28$ & $P<0.05$ \\
\hline Fish (3) & 0.63 & 3.39 & $1 \cdot 11$ & 3.34 & $1 \cdot 12$ & 3.45 & $1 \cdot 10$ & NS \\
\hline Dairy foods (7) & 0.75 & $3 \cdot 27$ & 0.87 & $3 \cdot 26$ & 0.81 & 3.27 & 0.93 & NS \\
\hline Vegetables (17) & 0.89 & 3.05 & 0.83 & $3 \cdot 13$ & 0.80 & 2.96 & 0.86 & $P<0.01$ \\
\hline
\end{tabular}

* Response scale for each item is $1-5$ with $1=$ 'I hate it' to $5=$ 'I love it'.

\section{Discussion}

Our results indicate that the ten most highly rated foods in this large sample of children in London were very similar to those cited in previous studies of French, German, American and younger British children (Diehl, 1999; Bellisle et al. 2000; Wardle et al. 2001; Skinner et al. 2002), with fatty \& sugary foods featuring most heavily. Nevertheless, the inclusion of two fruit items (grapes and strawberries) in the children's 'Top 10' is encouraging and unexpected. Vegetables, on the other hand, were over-represented in the lowest rated foods. Again, this is a widely replicated finding cross-culturally (Ton $\mathrm{Nu}$ et al. 1996; Gibson et al. 1998; Diehl, 1999; Skinner et al. 2002; PerezRodrigo et al. 2003) and to some extent explains the low levels of consumption typically reported. The findings on fruit, however, seem to challenge the idea that children eat what they like since we know that, on average, their fruit intake is low, although the high rating given to grapes and strawberries may reflect their status as 'treat' foods. These findings, together with previous studies of children's fruit and vegetable intake, emphasise the need to consider them as separate variables (Gibson et al. 1998; Cooke et al. 2004).

We have replicated the finding of Wardle et al. (2001) of a strong correlation between the average liking for a food and the proportion of children who had tried that food. As the authors point out, the latter is not a measure of exposure frequency, but the association suggests that parents tend to offer their children the foods that they accept most readily. Skinner et al. (2002) found that mothers were unlikely to introduce their children to foods that they themselves disliked, and this is reflected in our finding that the least tried foods were those that are widely disliked by adults (e.g. sprouts and liver).

As expected, the number of foods tried increased significantly with age in our sample, as did the number of foods liked and disliked. However, controlling for the number of foods tried eliminated the age-related increase in number of foods disliked, indicating that this was merely an artefact of having more foods to rate. More intriguing was the impact on number of foods liked, which was found to decrease with age as a function of the number tried. These findings might be considered to be support for the notion of a deterioration in the diets of older children (Lytle et al. 2000) rather than the broadening of dietary repertoire that a developmental decline in neophobia might lead us to expect (Pelchat \& Pliner, 1995; Koivisto \& Sjoden, 1996). One possible interpretation of these results is that the foods that children try later in life are intrinsically less likeable than those commonly offered to younger children. Thus, although a lessening of neophobia appears to increase the willingness to try new foods, it does not follow that a liking for these more 'acquired tastes' will result.

Research into the efficacy of repeated exposure in increasing liking for unfamiliar foods typically uses appealing new foods such as cheese and fruit (Birch \& Marlin, 1982) or fruit juice (Pliner, 1982). Where more unusual foods such as dates (Birch, $1979 a$ ) or tripe, halva and chutney (Peryam, 1963) have been the target, preference has not significantly increased. Alternatively, it is possible that levels of exposure in these studies were too low to have an impact. This may have been a factor in our findings, but we have no means of knowing the extent of participants' exposure to foods, merely that they had tried them

Table 2. Liking for items in each food category by age group (Mean values with their standard deviations)

\begin{tabular}{|c|c|c|c|c|c|c|c|c|c|}
\hline & \multicolumn{2}{|c|}{ Infants } & \multicolumn{2}{|c|}{ Juniors } & \multicolumn{2}{|c|}{$\begin{array}{l}\text { Lower second- } \\
\text { ary }\end{array}$} & \multicolumn{2}{|c|}{ Mid secondary } & \multirow[b]{2}{*}{ Significance of difference } \\
\hline & Mean & SD & Mean & SD & Mean & SD & Mean & SD & \\
\hline Fruits & $4 \cdot 18$ & 0.74 & $4 \cdot 28$ & 0.73 & $4 \cdot 11$ & 0.77 & $4 \cdot 17$ & 0.77 & $P<0.05$ \\
\hline Vegetables & $3 \cdot 15$ & 0.86 & 3.00 & 0.88 & 2.98 & 0.81 & $3 \cdot 11$ & 0.79 & NS \\
\hline Processed meat & 3.60 & 0.90 & 3.68 & 0.86 & 3.64 & 0.81 & $3 \cdot 72$ & 0.79 & NS \\
\hline Meat & 3.72 & 0.82 & 3.66 & 0.82 & 3.72 & 0.78 & 3.76 & 0.77 & NS \\
\hline Fish & $3 \cdot 71$ & 1.08 & $3 \cdot 34$ & $1 \cdot 18$ & $3 \cdot 36$ & 1.08 & 3.30 & 1.07 & $P<0.05$ \\
\hline Eggs & 3.55 & $1 \cdot 39$ & 3.46 & 1.33 & 3.56 & $1 \cdot 17$ & 3.55 & $1 \cdot 24$ & NS \\
\hline Fatty/sugary & $4 \cdot 29$ & 0.61 & $4 \cdot 41$ & 0.54 & $4 \cdot 23$ & 0.55 & $4 \cdot 19$ & 0.55 & $P<0.001$ \\
\hline Dairy & 3.47 & 0.89 & $3 \cdot 30$ & 0.99 & $3 \cdot 22$ & 0.80 & $3 \cdot 22$ & 0.81 & $P<0.005$ \\
\hline Starchy staples & $4 \cdot 07$ & 0.68 & 3.99 & 0.62 & 3.99 & 0.54 & 3.97 & 0.51 & NS \\
\hline
\end{tabular}


once or more. It is unlikely that participants would have tasted new foods the fifteen or more times required to change preferences.

There were no gender differences in number of foods tried, liked or disliked, although the significant age-by-gender interaction in number of foods disliked suggests that boys may be more 'picky' early, where girls are more so during adolescence. Although Koivisto-Hursti \& Sjoden (1997) have previously documented significantly higher neophobia in 9-year-old boys and in adult males, no gender differences were found in adolescents. An alternative explanation for girls disliking more foods may be that weight and diet issues become more pertinent for girls at this age, although this is speculative since we have no supporting data available for our sample.

Grouping foods into nine categories provided a more detailed picture of gender differences, changes in preferences that occur over time and the relationships between foods. Cronbach's alpha for all categories were high, indicating that liking was consistent within categories. Across age groups and genders, fatty \& sugary foods were liked the most and vegetables the least, which is largely consistent with previous findings (Ton Nu et al. 1996; Diehl, 1999; Skinner et al. 2002), although it should be noted that even among vegetables, average ratings were above indifference. Liking for fruit ran a close second to that for fatty \& sugary foods yet, as previously noted, intake is typically low. This apparent contradiction may be a product of the context in which fruit is generally offered - as a snack in direct competition with the more desirable options of sweets or salty snacks. This is a contest that fruit is unlikely to win given its low energy density and lesser sweetness.

Previous research has documented healthier food choices and greater liking and consumption of fruits and vegetables in girls when compared with their male peers (Reynolds et al. 1999; Le Bigot Macaux, 2001; Lien et al. 2001; Robinson \& Thomas, 2004), and our data show a similar pattern. Girls had a greater liking for fruit and vegetables than did boys, and boys gave higher ratings to fatty \& sugary foods, meat, processed meat and eggs than did girls. Given that at all ages, boys' energy requirements are greater than those of girls, their greater liking for more energy-dense food groups may serve an adaptive purpose. On the other hand, social desirability may have had a stronger impact on girls' responding, because of the greater importance that females attach to diet (Wardle et al. 2004).

The age differences in preferences for food categories observed here do not provide a wholly coherent picture. Significant differences were found in only four categories (fatty \& sugary, fruit, fish and dairy) and fail to support a prediction of an increased preference for unhealthy foods at or around puberty and the transition to secondary school. On the contrary, our data suggest a reduced preference for fatty \& sugary foods at this time, albeit combined with a similar reduction in liking for fruit. Likewise, age-by-gender interaction effects were highly variable and problematic to interpret.

We acknowledge some limitations of our study. First, the crosssectional nature of our data limits the implications that can be drawn. Clearly, there is a need to investigate food preferences longitudinally across this age span in British children. Second, the generalisability of our findings is questionable given that only six schools agreed to take part, two of which were single-sex. We were unable to adjust our analyses for an effect of school since this was confounded with age. This is regrettable since the individual school's food environment may have an influence on preferences. In addition, the fact that parents had a major part in completing questionnaires for the youngest of our age groups may have affected our results. Finally, it would have been informative to have more detail about the children themselves (e.g. ethnicity, socio-economic status, temperament and dietary restrictions) and their parents in order to investigate effects of these on preferences. Nevertheless, the large sample size and high coverage rate achieved lend weight to our findings.

Our aim was to investigate age and gender differences in food preferences in order to inform interventions to improve children's diets. Across ages and genders, children rate fatty $\&$ sugary foods very highly and, with a food industry all too eager to produce foods that appeal to their tastes, it is perhaps not surprising that we are facing an obesity epidemic. A developmental decrease in the number of foods liked may further counteract efforts to establish healthier diets in adolescents. As well as documenting the problem, however, our findings point to a number of possible areas for intervention.

Our results emphasise the strong relationship between familiarity and preferences. Effective strategies to increase consumption should therefore include elements designed to provide repeated exposure to unfamiliar foods. Findings for fruit and vegetables are also informative. The data suggest that dislike is not a barrier to fruit consumption since fruit was rated very highly, especially among girls. In contrast, our findings support the view that liking should be targeted in interventions aimed at increasing the consumption of vegetables, which are rated only slightly above indifference. The high consistency of preferences within categories might suggest that increasing the liking for one food in a category might generalise to liking for others in the same category. Finally, it appears that boys have less healthful food preferences than girls at almost every age, and interventions should be mindful of the need to target messages appropriately.

\section{Acknowledgements}

We thank David Boniface for statistical advice. L. C. and J. W. were responsible for the design of the study, data analysis and interpretation, and for writing the manuscript. L. C. was also responsible for collecting all the data. The Health Behaviour Unit at University College London is funded by Cancer Research UK.

\section{References}

Baxter SD \& Thompson WO (2002) Fourth-grade children's consumption of fruit and vegetable items available as part of school lunches is closely related to preferences. J Nutr Educ Behav 34, 166-171.

Bellisle F, Rolland-Cachera M-F \& Kellogg Scientific Advisory Committee 'Child and Nutrition' (2000) Three consecutive (1993, 1995, 1997) surveys of food intake, nutritional attitudes and knowledge, and lifestyle in 1000 French children, aged 9-11 years. J Hum Nutr Diet 13, $101-111$.

Birch LL (1979a) Dimensions of preschool children's food preferences. J Nutr Educ 11, 77-80.

Birch LL (1979b) Preschool children's food preferences and consumption patterns. J Nutr Educ 11, 189-192.

Birch LL (1999) Development of food preferences. Annu Rev Nutr 19, $41-62$. 
Birch LL \& Marlin DW (1982) I don't like it; I never tried it: effects of exposure on two-year-old children's food preferences. Appetite 3, 353-360.

Cooke L, Wardle J, Gibson EL, Sapochnik M, Sheiham A \& Lawson M (2004) Demographic, familial and trait predictors of fruit and vegetable consumption by preschool children. Publ Health Nutr 7, 295-302.

Diehl JM (1999) [Food preferences of 10- to 14-year-old boys and girls]. Schweiz. Med Wochenschr 129, 151-161.

Drewnowski A (1997) Taste preferences and food intake. Annu Rev Nutr 17, 237-253.

Garrett BE \& Griffiths RR (1998) Physical dependence increases the relative reinforcing effects of caffeine versus placebo. Psychopharmacology (Berlin) 139, 195-202.

Gibson EL, Wardle J \& Watts CJ (1998) Fruit and vegetable consumption, nutrition knowledge and beliefs in mothers and children. Appetite 31, 205-228.

Koivisto UK \& Sjoden PO (1996) Food and general neophobia in Swedish families: parent-child comparisons and relationships with serving specific foods. Appetite 26, 107-118.

Koivisto Hursti U-K \& Sjoden PO (1997) Food and general neophobia and their relationship with self-reported food choice: familial resemblance in Swedish families with children of ages 7-17 years. Appetite 29, 89-103.

Le Bigot Macaux A (2001) Eat to live or live to eat? Do parents and children agree? Public Health Nutr 4, 141-146.

Lien N, Lytle LA \& Klepp KI (2001) Stability in consumption of fruit, vegetables and sugary foods in a cohort from age 14 to age 21 . Prevent Med 33, 217-226.

Lytle LA, Seifert S, Greenstein J \& McGovern P (2000) How do children's eating patterns and food choices change over time? Results from a cohort study. Am J Health Promot 14, 222-228.

Nicklaus S, Boggio V, Chabanet C \& Issanchou S (2004) A prospective study of food preferences in childhood. Food Qual Prefer 15, 805-818.
Pelchat ML \& Pliner P (1995) 'Try it. You'll like it'. Effects of information on willingness to try novel foods. Appetite 24, 153-166.

Perez-Rodrigo C, Ribas L, Serra-Majem L \& Aranceta J (2003) Food preferences of Spanish children and young people: the enKid study. Eur J Clin Nutr 57, Suppl. 1, S45-S48.

Peryam DR (1963) The acceptance of novel foods. Food Technol 17, 33-39.

Pliner P (1982) The effects of mere exposure on liking for edible substances. Appetite 3, 283-290.

Pliner P \& Pelchat M (1986) Similarities in food preferences between children and their siblings and parents. Appetite 7, 333-342.

Resnicow K, Davis-Hearn M, Smith M, Baranowski T, Lin LS, Baranowski J, Doyle C \& Wang DT (1997) Social-cognitive predictors of fruit and vegetable intake in children. Health Psychol 16, 272-276.

Reynolds KD, Baranowski T, Bishop DB, Farris RP, Binkley D, Nicklas TA \& Elmer PJ (1999) Patterns in child and adolescent consumption of fruit and vegetables: effects of gender and ethnicity across four sites. J Am Coll Nutr 18, 248-254.

Robinson CH \& Thomas SP (2004) The interaction model of client health behavior as a conceptual guide in the explanation of children's health behaviors. Public Health Nurs 21, 73-84.

Skinner JD, Caruth BR, Wendy B \& Ziegler PJ (2002) Children's food preferences: a longitudinal analysis. J Am Diet Assoc 102, 1638-1647.

Sweeting H, Anderson A \& West P (1994) Socio-demographic correlates of dietary habits in mid to late adolescence. Eur J Clin Nutr $\mathbf{4 8 ,}$ 736-748.

Ton Nu C, MacLeod P \& Barthelemy J (1996) Effects of age and gender on adolescents' food habits and preferences. Food Qual Prefer 7, 251-262.

Wardle J, Haase AM, Steptoe A, Nillapun M, Jonwutiwes K \& Bellisle F (2004) Gender differences in food choice: the contribution of health beliefs and dieting. Ann Behav Med 27, 107-116.

Wardle J, Sanderson S, Gibson EL \& Rapoport L (2001) Factor-analytic structure of food preferences in four-year-old children in the UK. Appetite 37, 217-223. 